\title{
A Tractarian Universe
}

\author{
Albert Visser
}

Received: 30 July 2010 / Accepted: 17 February 2011 / Published online: 12 March 2011

(C) The Author(s) 2011. This article is published with open access at Springerlink.com

\begin{abstract}
In this paper we develop a reconstruction of the Tractatus ontology. The basic idea is that objects are unsaturated and that Sachlagen are like molecules. Bisimulation is used for the proper individuation of the Sachlagen. We show that the ordering of the Sachlagen is a complete distributive, lattice. It is atomistic, i.e., each Sachlage is the supremum of the Sachverhalte below it. We exhibit three normal forms for Sachlagen: the bisimulation collapse, the canonical unraveling and the canonical bisimulation collapse. The first of these forms is unique modulo isomorphism, the second and third are simply unique. The subset ordering on normal forms of the second and third kind reflects the ordering of the Sachlagen.
\end{abstract}

Keywords Wittgenstein • Tractatus • Ontology • Bisimulation • Unraveling

\section{Introduction}

Unsaturatedness is the hallmark of Tractarian objects. This, at least, is the interpretation of Tractatus that we will pursue in this paper. We develop a model of the Tractatus ontology, in which occurrences of unsaturated objects click

Dedicated to Roel de Vrijer on the occasion of his 60th birthday.

\footnotetext{
A. Visser $(\varangle)$

Department of Philosophy, Utrecht University, Janskerkhof 13a, 3512 BL Utrecht, The Netherlands

e-mail: albert.visser@phil.uu.nl
} 
together to form saturated Sachlagen and Sachverhalte. ${ }^{1}$ The Sachverhalte are atomic Sachlagen - in a sense of atomic that will be explicated.

An important point of the paper is the analysis of sameness of Sachlagen. This is an issue that Wittgenstein did not address, probably because he never attempted to build a concrete model of his Logical Space. As will be explained in the paper we opt for bisimularity as the appropriate analysis of sameness. This means, very roughly, that two Sachlagen are the same as they cannot be distinguished from an internal structural point of view.

\subsection{Saturated Versus Unsaturated}

Frege insisted that only in the context of a sentence does a word have meaning (the context principle). Now consider the sentence Roel is happy. We can view the predicate expression is happy as obtained by removing the name Roel from the sentence. So the predicate expression really is: ( ) is happy. We can now see that the predicate expression is ergänzungsbedürftig or unsaturated: it needs supplementation to form an expression that is meaningful in its own right, to wit the sentence. This Ergänzungsbedürftigkeit is, in Frege's set up, inherited on the semantic level by the predicate itself.

But what about referring expressions? What about Roel? It seems to me that if we were really serious about the context principle, we should say that Roel was really Roel ( ), and consequently that the object corresponding to the name is unsaturated. If we forget philosophy of language for a moment, we could say that, in this view, properties and relations can only have reality in the objects having them, but, conversely, that objects also only have reality in the properties they have and in the relations they stand in. Such a view would blur the distinction between object and property/relation.

The tradition has refrained from taking the step towards unsaturated objects. E.g., in Montague Grammar, we have a basic type of objects and a basic type of propositions/truth values. All other types are derived from these. The objects of the basic types can be viewed as the saturated entities in this framework: these are the entities that are not functions.

Wittgenstein, in my reconstruction, insisted that objects are ergänzungsbedürftig too. This is reflected in the fact that they do not occur independently. Here is a telling quote: ${ }^{2}$

2.0131 Der räumliche Gegenstand muß im unendlichen Raume liegen. (Der Raumpunkt ist eine Argumentstelle.) Der Fleck im Gesichtsfeld muß zwar nicht rot sein, aber eine Farbe muß er haben: er hat sozusagen den Farbenraum um sich. Der Ton muß eine Höhe haben, der Gegenstand des Tastsinnes eine Härte, usw.

\footnotetext{
${ }^{1}$ I will leave "Sachlage" and "Sachverhalt" untranslated. They are Tractarian expressions with very special meanings. Still, the Pearce \& McGuiness translation of "Sachlage" as situation, and of "Sachverhalt" as state of affairs, does reflect my interpretation of these words reasonably well.

${ }^{2}$ We use the Pearce \& McGuiness translation [9] throughout the paper.
} 
2.0131 A spatial object must be situated in infinite space. (A spatial point is an argument-place.) A speck in the visual field, though it need not be red, must have some colour: it is, so to speak surrounded by colour-space. Notes must have some pitch, objects of the sense of touch some degree of hardness, and so on.

In short, happiness cannot occur without someone being happy, but Roel cannot occur without being in some mood.

This reading finds some support in the exegetic literature. For example, here is the discussion by Max Black, in his classical [2], p 13. He says:

Frege took a decisive step forward when he introduces a radical distinction between 'functions' and 'objects'. The former being 'incomplete', 'in need of supplementation', 'unsaturated', have to be symbolized by words of a peculiar and distinctive sort ('function names'), having 'gaps' that need top be filled by names of objects. Wittgenstein went still further: for him, it might be said, all simple symbols (names) were unsaturated in something like Frege's sense. Names occur only in association with other names, and have no reference except in the propositional context (3.3); in elementary propositions they grip one another, without intermediaries, like links of a chain (cf. 3.14); and their esse is to be eligible for such concatenation $(3.203+3.21+2.0123)$.

We note that Black is discussing the unsaturatedness of names, not of objects. We take the unsaturatedness of names to be reflected in the unsaturatedness of objects, or, if you wish, the unsaturatedness of names to reflect the unsaturedness of objects. (Black refers to 2.0123 which is about objects.)

The above discussion takes our ordinary understanding of world and language as starting point and reasons from there about what the tractarian objects could be. If we start from the standpoint of Wittgenstein's ontology, we just have unsaturated objects. ${ }^{3}$ There are no relations and traditional objects. Since Tractarian objects are not given to us in 'surface language', we could easily imagine that for some of these objects it is impossible to say whether they are more like traditional objects or traditional relations. They simply need not have traditional counterparts.

Remark 1.1 Nominalism versus Realism: There has been some discussion whether universals like $R$ are objects in Wittgenstein's sense. Do they occur as objects in configurations, or do they subsist in the way objects click together? The positive answer to this question can be labeled realism, the negative answer nominalism.

Tractatus 3.1432 seems to speak against the objectual understanding of universals. On the other hand, our leading idea of a rapprochement between objects-qua-particulars and universals because they are all just unsaturated, strongly suggests an equal treatment of $a$ and $R$.

${ }^{3}$ I thank Jesse Mulder for stressing this point to me. 
We have already stressed that the question concerning traditional objects and relations, does not arise when we start from the ontology. Since our framework just models the ontology, it is neutral between realism versus nominalism. However, the motivation of our framework, looking from language to ontology, fits better with the realist option.

It is more clumsy to interpret familiar patterns of predication nominalistically. Consider, e.g., Sachverhalte $\left[P_{0} a\right],\left[P_{1} a\right], \ldots$, of the traditional subject-predicate form, where the $P_{i}$ are supposed to be different. If in the Sachverhalte nothing corresponds with the $P_{i}$, the differences between the Sachverhalte must be accounted for as different ways in which $a$ is linked to itself. For example, we could suppose that $a$ has two poles $p, q$, where both poles may click together both with themselves and with each other. ${ }^{4}$ This would give us two possible Sachverhalte only involving one occurrence of $a$ : one where each pole clicks with itself en one where the poles click with each other. More generally an object that has $n$ poles that can click together both with themselves and with each other, can form a one-object Sachverhalt in $n$ ! ways. So there are precisely $n$ ! unary predicates applicable to $a$. Of course, we can make the different poles of $a$ of different types, so that more interesting patterns of clicking together become possible. Thus, we can raise the number of possible properties of $a$ by working with more poles that fit together. All this seems rather unattractive. It feels like a hack.

See for a discussion of exegetical matters concerning the nominalism versus realism issue, e.g., the chapter on Problèmes ontologiques in [7], pp. 68-84.

\subsection{Objects in Multiplicity}

How precisely do the objects form a chain or configuration? We will assume that configurations are not set-like. If they were set-like, how could we explain the difference between $[a R b]$ and $[b R a]$ ? They are more like molecules where the objects are connected via links or poles.

Consider a Sachverhalt of the form [aRa]. Wittgenstein's discussion of identity could carry some suggestion that he would not allow configurations of this form. However, there is a powerful reason not to disallow such configurations, to wit intersubstitutivity of objects of the same logical form in Sachverhalte. The notion of logical form does not necessarily imply a substitution principle, but it certainly does cohere well with a substitution principle. We would like to demand the following: if $a$ and $b$ have the same logical form, then we can always substitute $a$ for $b$ in a configuration. Thus $[a R a]$ can be 'derived' from $[a R b]$. How can we think about $[a R a]$ ? One idea is that $a$ has two poles, say $p$ and $q$. The pole $p$ combines with the first pole of $R$, the pole $q$ combines with the second pole of $R$. Saturatedness of [aRa] dictates that $a$ does not have more poles. But then consider $[P a]$. Here $a$ can have only one pole. Should we

\footnotetext{
${ }^{4}$ We say that the objects are connected via poles. This will be discussed in more detail in Section 1.3.
} 
allow $a$ to have a varying number of poles? But if this number varies, it should vary when we move from $[a R a]$ to $[P a]$, so what varies here to make $a$ 'change' its number of poles?

We consider a variant of our example: presumably both $[a R b]$ and $[P a]$ could be Sachverhalte. Since they are supposed to be saturated, in each case, $a$ has exactly one pole. However, how can this pole be employed twice? This phenomenon can be explained by the insight that what we find in Sachverhalte are occurrences of objects. We need not stipulate a varying number of poles to account for the possibility of both $[a R a]$ and $[P a]$ : we can say that $a$ has two occurrences in $[a R a]$. We note there is some textual evidence that Wittgenstein thought about objects as occurring in states of affairs. For example, in 2.012, he says:

2.012 In der Logik ist nichts zufällig: Wenn das Ding im Sachverhalt vorkommen kann so muß die Möglichkeit des Sachverhaltes im Ding bereits präjudiziert sein.

2.012 In logic nothing is accidental: if a thing can occur in a state of affairs, the possibility of the state of affairs must be written into the thing itself.

Thus, we will model our logical spaces by implementing a notion of occurrence of an object. As we will see, in our framework, occurrences have very thin identities. Not only are they like indiscernibles, but, in many examples, even their cardinality is indeterminate.

\subsection{How Objects Click Together}

The poles are what connects objects to each other. Each pole connects to another pole. Not all poles may click together. Each pole has a type. Ony poles with types that match may connect. Which types match is given with the specification of logical space.

We will assume that the poles of an object are fixed. This is assumed just for the sake of simplicity. We see no knock-down philosophical argument against the possibility of a varying number of poles for some objects or relations. This would give us 'polyadic objects'. Also, at first sight, it seems pretty straightforward to generalize our framework to accommodate variable sets of poles. ${ }^{5}$

We will also assume that connection is always a matter of $t w o$ poles. ${ }^{6}$ Again this is just an assumption for the sake of simplicity. The framework can easily be adapted to multi-linking.

Finally, we will assume that our poles have individuality. They are not indiscernibles. The reason for this choice is the intuition that the neatest way

\footnotetext{
${ }^{5}$ Varying assemblies of poles are suggested as one possible option for obtaining a more systematic view of objects as special cases of (possibly) unsaturated configurations. See Section 5, for further remarks on this matter.

${ }^{6}$ This assumption makes our Sachlagen into linear graphs. See Section 1.6, for a reference.
} 
to analyse a first-order style predication like $[P a b]$, by giving $P$ two poles of type, say, $a$ and $a$ and $b$ each one pole of type, say, b. Here a and b match. In Example 2.7, this idea is worked out. So the argument places are given by the individuality of the poles; they are not coded in the types. Again it is easy to adapt the framework to have indiscernible poles. E.g., if all poles were indiscernible, the poles associated to an object would be a multiset of types.

\subsection{Sachlagen and Sachverhalte}

Sachlagen are like molecules. They are saturated configurations of objects which are clicked together via their poles in the ways that are allowed by the nature of logical space. Sachlagen are the primary candidates for existence.

Sachverhalte are special Sachlagen. In the framework they are the smallest non-empty Sachlagen. A Sachlage is not a set of Sachverhalte, but as we will see it is fully determined by a set of Sachverhalte.

There is an important interpretive choice in our treatment of Sachlagen. A Sachlage is a (possible) situation that is depicted in a picture. It is neutral with respect to existence. An existing Sachlage is a Tatsache or fact. According to our interpretation, there are no disjunctive pictures. So a sentence of the form $p \vee q$, where $p$ and $q$ are atomic does not depict. Its interpretation is handled by the truthfunctional semantics, which sits on top of the picture theory.

Sachlagen appear in two roles. We can take them as presenting something of the form at least this is the case (inclusive role) or as presenting this is the case and it is all that is the case (exclusive role). In their inclusive role, Sachlagen are parts of possible worlds and, in their exclusive role, they are full possible worlds. The actual world is the existing Sachlage in its exclusive role.

A Sachlage in the in the inclusive role corresponds to a conjunction of atomic sentences, and a Sachlage in the exclusive role corresponds to a conjunction of atomic sentences and negated atomic sentences (together: atomic literals) in which all possible atomic sentences occur precisely once.

Remark 1.2 We remind the reader of what Wittgenstein says in 2.11:

2.11 Das Bild stellt die Sachlage im Logischen Raume, das Bestehen und Nichtbestehen von Sachverhalten, vor.

2.11 A picture presents a situation in logical space, the existence and nonexistence of states of affairs.

This is somewhat mysterious, because Wittgenstein includes the nonexistence of Sachverhalten as something that is depicted. Maybe the existing Sachverhalte are in black and blue and red and the non-existing ones in yellow and pink and sepia? Such a subtle convention, surely would be beyond the range of simple depiction?

In an alternative reading, Wittgenstein takes the exclusive reading as the default. Thus, what is depicted would always be the Sachlage in its exclusive role. In other words, pictures are always full pictures of possible worlds. This 
reading would solve how non-existence can be pictured, however, it diverges substantially from the ordinary understanding of picture. Moreover, it seems improbable that even Wittgenstein would have such a subtle reading of what a picture does, without commenting upon it.

It would fit my interpretation better if Wittgenstein had omitted the phrase about non-existence.

The objects that we produce in our modeling to represent Sachlagen are neutral with respect to inclusive or exclusive role. A Sachlage will simply be a saturated configuration of objects.

Between Sachlagen (in the inclusive role) we have the sub-Sachlage partial ordering. A Sachverhalt is an atom in this ordering. This means that a Sachverhalt is a minimal non-empty Sachlage.

We will prove the theorem that Wittgenstein used but failed to state: the sub-Sachlage ordering is atomistic, i.e., every Sachlage is the supremum of the Sachverhalte below it.

Remark 1.3 The framework allows that there are saturated objects. This shows that it is conceivable that at least some objects are, or, at least, coincide with Sachlagen. Also both Sachlagen and objects belong to an encompassing category, say, the configurations. ${ }^{7}$ For more on configurations, see Section 5.

\subsection{When Two Sachlagen are the Same}

In the present paper, we are constructing models of logical spaces against the background of a classical meta-theory or modeling medium. As a modeling medium we employ informal set theory, say, with urelements for items like objects and poles. We are assuming that the urelements form a set, that the poles of an object form a set, etcetera. The objects we provide to represent basic items like Sachverhalte and Sachlagen are supposed to represent Sachverhalte and Sachlagen as they are philosophically intended, but there is no claim that the items constructed are Sachverhalte and Sachlagen. What is more the objects we construct contain artifacts of the modeling, aspects that are not present in the Sachverhalte and Sachlagen as philosophically intended.

One important matter is the question of sameness of two (models of) Sachverhalte. Consider, for example, an object $a$ with two poles $p$ and $q$ of type, respectively, a and $\mathrm{b}$. Suppose $\mathrm{a}$ and $\mathrm{b}$ match. In this case, we could have one occurrence of $a$ that links with itself. We could also have two occurrences of $a$, such that each links with the other. Or we could have three occurrences of $a$ that link in a circle. We could have an infinity of occurrences of $a$ that link with the order type of the integers. Are all these possibilities the same or different?

\footnotetext{
${ }^{7}$ Of course, we could hold that an object is conceptually different from the configuration containing just that object. In fact, I think that is the best way of looking at it.
} 
My intuition about this question can best be explained by introducing the idea of the inner perspective on a Sachlage. Suppose we had a fairy living in a Sachlage. What would that be like? The fairy would be at an occurrence of an object and would be able to travel across the poles to other occurrences. She would be able to identify those things that have true individuality: the object where she currently is, the link across which she travels. She would have no access to the identity of the occurrence: the occurrence is 'thin', it is just a 'multiplicity'. We may compare this idea with the intrinsic notion of curvature in a geometry that can be understood independently of an embedding space.

My idea is that two Sachlagen are the same if they are the same from the inner perspective, or even stronger: there is nothing to the Sachlage than the inner perspective. The outer perspective only exists as an artifact of the modeling.

Consider two Sachlagen $\sigma$ and $\tau$. Consider any object-occurrence $(d, i)$ in $\sigma$ and any object-occurrence $(e, j)$ in $\tau$. Suppose we drop fairy Femke in $(d, i)$ and fairy Inge in $(e, j)$. We suppose that Femke and Inge can communicate by mobile phone. We say that $(d, i)$ and $(e, j)$ are internally indistinguishable or observationally equivalent, if there is no way that Femke and Inge can find a difference between them by moving from object occurrence to object occurrence, carefully noting along which poles they travel. So, Inge and Femke try out paths through their respective Sachlagen and check whether they can find differences. If they cannot possibly find differences in this process, the initial occurrences are internally indistinguishable. Of course, indistinguishability implies that $d=e$.

We note that Femke and Inge are not allowed to leave breadcrumbs. Leaving breadcrumbs would change the Sachlage, which is impossible-even for a fairy.

The Sachlagen $\sigma$ and $\tau$ are the same if, for every object-occurrence $(d, i)$ in $\sigma$, there is an internally indistinguishable object-occurrence $(d, j)$ in $\tau$, and vice versa.

Our intended notion of sameness can be technically explicated using the well established notion of bisimilarity. This notion is employed in Computer Science. For example, in Process Algebra it is used to explicate sameness of processes. It is also employed in Modal Logic to provide a notion of sameness of Kripke models. It and related concepts provide a good tool for proving various theorems. The application of bisimulation that is, perhaps, closest to the use we are making of it in this paper, is the analysis of non-wellfounded sets. We refer the reader to Peter Aczel's wonderful book [1]. ${ }^{8}$

Remark 1.4 There is some abus de langage, in that we call representations of Sachlagen "Sachlagen", and representations of Sachverhalte "Sachverhalte". It is my feeling that the alternative of using different names, say "pre-

\footnotetext{
${ }^{8}$ For a discussion of individuation of sets, see also Adam Rieger's eminently readable paper [8]. Unfortunately, the Rieger paper does not have so much emphasis on notions of bisimulation.
} 
Sachverhalte" for representations of Sachverhalte, is so unpleasant that the awkwardness of our usage is well worth the cost. So, we will use "Sachlage" and "Sachverhalt" for the items in our modeling, without dividing out bisimilarity. We will say things like when two Sachlagen are the same, meaning: when two representations of Sachlagen are bisimilar.

\subsection{Linear Graphs}

Vincent van Oostrom pointed out to me that my modeling of Sachlagen is very much similar to linear graphs as studied by Alan Bawden in his paper Implementing Distributed Systems using Linear Naming (see: http://dspace. mit.edu/handle/1721.1/7085).

Linear graphs turn out to be almost the same as (models of) Sachlagen. There are some minor differences. We may translate our concepts into Bawden's as follows.

\begin{tabular}{lll}
\hline This paper & Bawden's paper & Modern graph rewriting \\
\hline Object & Type & Type \\
Object-occurrence & Vertex & Vertex \\
Pole-occurrence & Terminal & Port \\
Pole & Label & - \\
Type & - & - \\
\hline
\end{tabular}

Under this translation, the poles in Bawden's framework are chosen from a fixed set. They may be viewed as all having the same type that clicks with itself.

Bawden also uses bisimulation as the proper relation of equality. His explanation of why this is a good choice is very much similar to mine. Since Bawden's study is from 1993, he has clear priority for a number of ideas.

Finally we note that, where Tractarian logical spaces are static, graph rewriting adds a dynamics and studies processes that modify linear graphs.

\subsection{Significance of this Paper}

I submit that the study of these logical spaces à la Wittgenstein, has some interest beyond the historical reconstructive question whether one can make sense of the ontology of the Tractatus. Even if precisely these spaces are rather clearly not a good model for natural language semantics, the idea of nonsaturated objects is a good one-and one that has not been studied sufficiently seriously. In this light, what we are doing here can be viewed as a study of unsaturated objects under radical simplifying assumptions.

In the previous subsection, we discussed the similarity between linear graphs and Sachlagen. This convergence also shows that we are looking at a natural idea. 


\section{Let There be Sachlagen}

In this section we develop our (class of) model(s) of the Tractatus universe. It is important to stress that we are not aiming at a foundational presentation, building up the Tractarian world and its metatheory in tandem. We simply employ the usual set theoretic metatheory, say ZF with urelements, and we assume that the ingredients of Wittgensteinian logical spaces can be taken to be just sets. It will then, e.g., be a theorem (Theorem 4.3) that the totality of Sachlagen can be represented by a set.

A logical space is given by:

- A non-empty set of objects $D$,

- A set of types $T$,

- A binary matching relation $M$ between types. We demand that $M$ is symmetric.

- A function $P$ that assigns to each object a (possibly empty) set of poles.

- A typing function $F$ on $D$ such that $F_{d}: P_{d} \rightarrow T$.

Every object comes equipped with a set of poles. Even if the precise identity of these poles is immaterial, they still are like fixed argument-places or argument-roles. Of course, a more flexible implementation would be possible, e.g. adding a group of permutations to the set of poles to make certain places 'the same', but we will refrain from adding such extra features in the present implementation. ${ }^{9}$ Each pole has a 'type'. The matching relation tells us which types may combine.

Two objects $d$ and $d^{\prime}$ have the same logical form if there is a type-preserving bijection between $P_{d}$ and $P_{d^{\prime}}$.

We turn to the definition of a Sachlage in a given logical space. We model occurrences by assigning to each object a set of indices. ${ }^{10}$ Thus, a Sachlage $\sigma$ is given by:

- A function $I$ from objects to (possibly empty) sets of indices.

- A binary relation $R$ on $O_{\sigma}^{\text {pole }}$, where $O_{\sigma}^{\text {pole }}$, the set of pole occurrences, is the set of $(d, i, p)$, where $i$ is in $I_{d}$ and $p$ is in $P_{d}$. We demand that $R$ is total (in the sense: $\forall x \exists y R x y$ ), functional and symmetrical and that $(d, i, p) R$ $\left(d^{\prime}, i^{\prime}, p^{\prime}\right)$ implies $F_{d}(p) M F_{d^{\prime}}\left(p^{\prime}\right)$.

\footnotetext{
${ }^{9}$ Such more refined treatments of argument-places are studied in the philosophy of neutral relations. See, e.g., $[4,5]$ and [6].

${ }^{10}$ This is a good point to note some disanalogies with the molecules of physics. Firstly, we can have different physical molecules of the same type. In contrast every Sachlage is unique. Secondly, in physics, the same atom (numerically) cannot occur in different molecules and it cannot occur a number of times in the same molecule (at the same time). Different atoms of the same type may occur in different molecules. A type of atom may occur a number of times in the same molecule. In contrast, a tractarian object may occur in several Sachlagen and may occur a number of times in the same Sachlage.
} 
The function $I$ gives us, for each object, the set of occurrences of that object. The set of occurrences of objects in a Sachlage $\sigma$ is $O_{\sigma}^{\mathrm{obj}}$, the set of pairs $(d, i)$, where $i \in I_{d}$. The relation $R$ tells us which poles click together in the Sachlage. We only allow poles to click together if their types match. The demand that $R$ is total embodies the idea that Sachlagen are saturated.

One pleasant alternative way to think about $R$ is as a set of arrows $\alpha$ between object-occurrences of the form $((d, i), p, q,(e, j))$. Here $(d, i)$ is the domain of $\alpha$ and $(e, j)$ is the codomain of $\alpha$. We represent such an arrow as $(d, i) \stackrel{p, q}{\longrightarrow}(e, j)$. We write $(d, i) \stackrel{p, q}{ }(e, j)$, if we want to stress the symmetry of $R$.

It is useful to also have a click relation at hand that ignores the poles via which occurences of objects are connected. Thus, we define:

- $\quad(d, i) \widetilde{R}\left(d^{\prime}, i^{\prime}\right) \quad: \Leftrightarrow \exists p, p^{\prime}(d, i, p) R\left(d^{\prime}, i^{\prime}, p^{\prime}\right)$.

Remark 2.1 We note that we allow a pole-occurrence to click with itself. This seems somewhat unnatural. It is like a hand that shakes itself. However, excluding it will complicate our notion of bisimulation collapse. Moreover, modulo bisimulation we can always replace such self-clicks by non-self-clicks. This happens for example in the canonical unraveling. See Section 4.

We turn to the definition of sameness of Sachlagen. We will identify as many occurrences as is reasonable. We identify all occurrences that we can consistently identify given that we want their environments to be similar. We say that $B$ is a bisimulation between $\sigma$ and $\sigma^{\prime}$ iff $B$ is a function on objects that assigns to each $d$ a relation $B_{d}$ between $I_{d}$ and $I_{d}^{\prime}$, such that $B_{d}$ satisfies the zigand the zag-property (see Fig. 1):

- If $i B_{d} i^{\prime}$ and $(d, i, p) R(e, j, q)$, then, for some $j^{\prime}$ in $I_{e}^{\prime}$, we have $j B_{e} j^{\prime}$ and $\left(d, i^{\prime}, p\right) R^{\prime}\left(e, j^{\prime}, q\right)$ (zig-property / forward property),

- If $i B_{d} i^{\prime}$ and $\left(d, i^{\prime}, p\right) R^{\prime}\left(e, j^{\prime}, q\right)$, then, for some $j$ in $I_{e}$, we have $j B_{e} j^{\prime}$ and $(d, i, p) R(e, j, q)$ (zag-property / backward property).

Note that, in an alternative presentation, we could view a bisimulation as a relation between the sets of object occurrences $O_{\sigma}^{\text {obj }}$ and $O_{\sigma^{\prime}}^{\text {obj }}$. So $B$ of our official definition would be represented by $B^{\circ}$ with:

- $(d, i) B^{\circ}\left(d^{\prime}, i^{\prime}\right): \Leftrightarrow d=d^{\prime}$ and $i B_{d} i^{\prime}$.

Fig. 1 The Zig and the Zag property

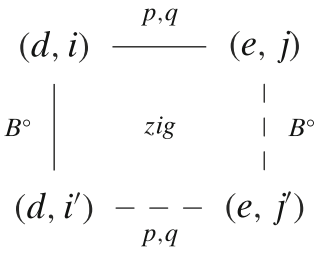


Fig. 2 Zig implies Zag

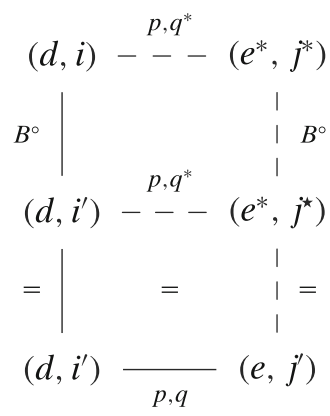

It is easy to regain $B$ from $B^{\circ}$. It will be pleasant to move in a flexible way between $B$ and $B^{\circ}$. The domain of $B$ will be the domain of $B^{\circ}$, and, similarly, for the range.

A bisimulation $B$ is total if, for each object $d$, the relation $B_{d}$ is total. It is surjective if, for each $d, B_{d}$ is surjective. The Sachlage $\sigma$ is a sub-Sachlage of $\sigma^{\prime}$, or $\sigma \preceq \sigma^{\prime}$, iff there is a total bisimilation $B$ from $\sigma$ to $\sigma^{\prime}$. We will write $B: \sigma \preceq \sigma^{\prime}$, if we want to exhibit the witnessing bisimulation $B$. The Sachlage $\sigma$ is equal to $\sigma^{\prime}$, or $\sigma \equiv \sigma^{\prime}$, iff there is a total and surjective bisimilation $B$ from $\sigma$ to $\sigma^{\prime}$. We will write $B: \sigma \equiv \sigma^{\prime}$, if we want to exhibit the witnessing bisimulation $B$.

Remark 2.2 A surprising aspect of our framework is that the zig property implies the zag property (and vice versa). Here is the argument. (See Fig. 2). Consider Sachlagen $\sigma$ and $\sigma^{\prime}$. Suppose we have the zig property from $\sigma$ to $\sigma^{\prime}$. Suppose $i B_{d} i^{\prime}$ and $\left(d, i^{\prime}, p\right) R^{\prime}\left(e, j^{\prime}, q\right)$. Since $\sigma$ is saturated, there must be $e^{*}, j^{*}, q^{*}$, such that $(d, i, p) R\left(e^{*}, j^{*}, q^{*}\right)$. By the zig property, we find that, for some, $j^{\star}$, we have $j^{*} B_{e^{*}} j^{\star}$ and $(d, i, p) R\left(e^{*}, j^{\star}, q^{*}\right)$. Since, $R$ is functional, it follows that $\left(e, j^{\star}, q^{*}\right)=\left(e, j^{\prime}, q\right)$. So, $j^{*}$ witnesses the backward property.

Each Sachlage $\sigma$ in its exclusive role represents a possible world. We impose the reasonable constraint that the existing Sachlagen (in their inclusive role) are closed under sums. We will show below that this implies that there is a maximal existing Sachlage.

When viewing $\sigma$ as a possible world, we say that $\sigma^{\prime}$ exists in $\sigma$, or $\sigma^{\prime}$ is a fact of $\sigma$, if $\sigma^{\prime} \preceq \sigma$.

Here are the basic facts about bisimulations.

\section{Theorem 2.3}

a. Let $\mathrm{id}_{\sigma}$ be given by: $\mathrm{id}_{\sigma, d}$ is the identity relation on $I_{\sigma, d}$. We have: $\mathrm{id}_{\sigma}$ is a total and surjective bisimulation from $\sigma$ to $\sigma$.

b. For any bisimulation $B$ from $\sigma$ to $\sigma^{\prime}$, let $\breve{B}$ be given by the stipulation that $\breve{B}_{d}$ is the converse relation of $B_{d}$, for each $d$. We have: $\breve{B}$ is a bisimulation from $\sigma^{\prime}$ to $\sigma$. 
c. Let $B$ be a bisimulation from $\sigma$ to $\sigma^{\prime}$ and let $B^{\prime}$ be a bisimulation from $\sigma^{\prime}$ to $\sigma^{\prime \prime}$. We define $B ; B^{\prime}$ by: $\left(B ; B^{\prime}\right)_{d}$ is the composition in the order of reading of $B_{d}$ and $B_{d}^{\prime}$. We have: $B ; B^{\prime}$ is a bisimulation.

d. Let $\mathcal{B}$ be a set of bisimulations from $\sigma$ to $\sigma^{\prime}$. We define $\cup \mathcal{B}$ as given by: $(\cup \mathcal{B})_{d}$ is the union of the $B_{d}$ for $B$ in $\mathcal{B}$. We find: $\cup \mathcal{B}$ is a bisimulation.

The proof of the theorem is entirely routine. The theorem gives us immediately the following corollary.

Corollary 2.4 The relation $\preceq$ is a partial pre-ordering with $\equiv$ as its induced equivalence relation.

Proof For example, if $B_{0}: \sigma \preceq \sigma^{\prime}$ and $B_{1}: \sigma^{\prime} \preceq \sigma$, then $\left(B_{0} \cup \breve{B}_{1}\right): \sigma \equiv \sigma^{\prime}$. Conversely, if $B: \sigma \equiv \sigma^{\prime}$, then $B: \sigma \preceq \sigma^{\prime}$ and $\breve{B}: \sigma^{\prime} \preceq \sigma$.

We define the empty Sachlage, $\Perp$, by: $I_{\Perp, d}:=\emptyset$, for all $d$ and $R_{\Perp}$ is the empty relation. Clearly, $\Perp$ is the minimal element w.r.t. of $\preceq$. Its $\equiv$-equivalence class of $\Perp$ contains precisely one element. We will show that there is also a maximal Sachlage $\pi$. See Theorem 3.11.

We define a Sachverhalt as an atom of $\preceq$. This means that $\sigma$ is a Sachverhalt iff, for any $\sigma^{\prime} \preceq \sigma$, we have $\sigma^{\prime} \equiv \sigma$ or $\sigma=\Perp$.

We end this section by presenting some examples.

Example 2.5 Consider the following logical space BASIC. We just have a single object ens. The object ens has no poles, i.o.w., $P_{\text {ens }}:=\emptyset$. The space has no types, and, consequently, the relation $M$ is empty. Our space has two Sachlagen $($ modulo $\equiv)$. First we have the empty Sachlage nil or $\Perp$ with $I_{\text {nil,ens }}=\emptyset$ and $R_{\text {nil }}$ is the empty relation. Then there is the Sachlage esse or $\pi$ in which ens is present. Here $I_{\text {esse,ens }}=\{0\}$ and $R_{\text {esse }}$ is the empty relation. Here esse is the only Sachverhalt of our space.

Example 2.6 Consider the following logical spaces $\mathcal{S}_{0}$ and $\mathcal{S}_{1}$. In both spaces we just have a single object $d$. In both spaces, we have $P_{d}:=\{p\}$. Both spaces have one type a. We take $M_{\mathcal{S}_{0}}:=\emptyset$ and $M_{\mathcal{S}_{1}}:=\{\langle a, a\rangle\}$.

Clearly $\mathcal{S}_{0}$ has only the empty Sachlage $\Perp$. The poor object $d$ is eternally blocked from participating is a Sachlage.

The space $\mathcal{S}_{1}$ has as Sachlagen $\Perp$ and a second Sachlage solo. Modulo isomorphism, solo has just two connected representations. The first representation is the following. The object $d$ has one occurrence, say 0 . The clickrelation is defined by: $R_{\text {solo }}=\{\langle(d, 0, p),(d, 0, p)\rangle\}$. The second representation looks as follows. The object $d$ has two occurrences, say 0 and 1 . We have $R_{\text {solo }}=\{\langle(d, 0, p),(d, 1, p)\rangle,\langle(d, 1, p),(d, 0, p)\rangle\}$. There are class-many other representations consisting of sums of disjoint copies of the ones just given.

In Section 4, we will introduce the bisimulation collapse and the canonical unraveling. Modulo isomorphism, our first representation is the bisimulation collapse and our second representation is the canonical unraveling. 
Example 2.7 Let some first order relational signature $\Sigma$ be given and some non-empty set of objects $X$. We assume that the relation symbols in $\Sigma$ and the objects in $X$ are disjoint. We take $D$ the union of $\Sigma$ and $X$. For a relation symbol $r$ of arity $n$, we take $P_{r}:=n:=\{0, \ldots, n-1\}$. For an object $x \in X$, we take $P_{x}:=1$. Let $F_{r, i}:=\mathrm{a}$ and $F_{x, 0}:=\mathrm{b}$. Let $M:=\{(\mathrm{a}, \mathrm{b}),(\mathrm{b}, \mathrm{a})\}$.

In this example the Sachverhalte correspond precisely with what we would take intuitively as Sachverhalte over the signature. E.g., the Sachverhalt rxy, where $r$ is binary, is modeled as $\sigma$, where, for $z \in X, I_{\sigma, z}=0$ if $z \neq x$ and $z \neq y$, and $I_{\sigma, z}:=1$, if $z=x$ or $z=y$. Moreover $I_{\sigma, s}=0$, if $s$ is a relation symbol not equal to $r$, and $I_{\sigma, r}:=1$.

\section{Example 2.8 In 4.2211, Wittgenstein writes:}

4.2211 Auch wenn die Welt unendlich complex ist, so daß jede Tatsache aus unendlich viele Sachverhalten besteht und jeder Sachverhalt aus unendlich vielen Gegenständen zusammengesetzt ist, auch dann müßte es Gegenstände und Sachverhalte geben.

4.2211 Even if the world is infinitely complex, so that every fact consists of infinitely many states of affairs and every state of affairs is composed of infinitely many objects, there would still have to be objects and states of affairs.

In this example, we show that one may imagine Sachverhalte that consist not of infinitely many objects, but of infinitely many occurrences of two objects. In our example we have an finite logical space with an infinite Sachverhalt. Consider a logical space with two objects $d$ and $e$. The first object $d$ has one pole, to wit $p$. The second object $e$ has two poles, to wit $q$ and $r$. The types are a and b. We set $F_{d} p:=\mathrm{a}, F_{e} q:=\mathrm{b}, F_{e} r:=\mathrm{a}$. We take $M:=\{(\mathrm{a}, \mathrm{b}),(\mathrm{b}, \mathrm{a})\}$. We now consider the Sachlage that is given as follows.

- $I_{d}:=\{*\}, I_{e}:=\omega$,

- The relation $R$ is generated by the following stipulations: $(d, *, p) R(e, 0, q),(e, 0, r) R(e, 1, q), \ldots,(e, n, r) R(e, n+1, q), \ldots$

Thus, our our Sachlage looks like this:

$$
(d, *) \stackrel{p, q}{\longrightarrow}(e, 0) \stackrel{r, q}{\longrightarrow}(e, 1) \stackrel{r, q}{-} \ldots(e, n) \stackrel{r, q}{-}(e, n+1) \ldots
$$

It is easily seen that this example indeed gives us a Sachlage (even a Sachverhalt) and that the maximal bisimulation of this Sachlage and itself is the identity, so the infinity of occurrences is 'real'.

We leave it as an exercise to the reader to construct an infinite Sachlage in a logical space with only one object. 


\section{The Ordering of the Sachlagen}

In this section we study the ordering $\preceq$. We show a.o. that it is, modulo $\equiv$, an atomistic, complete distributive lattice. The results of this section often have alternative proofs using the normal form theorems of Section 4.

\subsection{Basic Facts}

To develop some feeling for what a Sachlage looks like, we will first prove some basic facts. To formulate these we give some basic definitions.

- A set of object-occurrences $X$ is closed in $\sigma$, if, whenever $(d, i) \in X$ and $(d, i) \widetilde{R}_{\sigma}(e, j)$, then $(e, j) \in X$. If the Sachlage is clear from the context, we will simply say that $X$ is closed.

- Suppose $X$ is closed in $\sigma$. We define $\sigma \nmid X$ or $\sigma_{X}$ as follows.

- $I_{\sigma \mid X, d}:=\left\{i \in I_{\sigma, d} \mid(d, i) \in X\right\}$,

- $(d, i, p) R_{\sigma \mid X}\left(d^{\prime}, i^{\prime}, p^{\prime}\right): \Leftrightarrow(d, i, p) R_{\sigma}\left(d^{\prime}, i^{\prime}, p^{\prime}\right)$,

where $i \in I_{\sigma \mid X, d}$ and $i^{\prime} \in I_{\sigma \mid X, d^{\prime}}$.

Clearly, $\sigma_{X}$ is again a Sachlage.

- $\sigma \sqsubseteq \tau$ iff, for all $d, I_{\sigma, d} \subseteq I_{\tau, d}$ and $R_{\sigma} \subseteq R_{\tau}$.

- $E_{\sigma}$ is the transitive, reflexive closure of $\widetilde{R}_{\sigma}$. Clearly, $E_{\sigma}$ is an equivalence relation. We call the $E_{\sigma}$-equivalence class of $(d, i)$ in $O_{\sigma}^{\text {obj }}:[d, i]_{\sigma}$. A Sachlage $\sigma$ is connected if it has at most one $E_{\sigma}$-equivalence class. We write $\sigma[d, i]$ for $\sigma\left\lceil[d, i]_{\sigma}\right.$. It is easily seen that $\sigma[d, i]$ is a connected Sachlage.

- Suppose $B$ is a bisimulation between $\sigma$ and $\tau$ (not necessarily total or surjective). Suppose $\sigma^{\prime} \sqsubseteq \sigma$ and $\tau^{\prime} \sqsubseteq \tau$. We define $B_{\sigma^{\prime}, \tau^{\prime}}$ by $i B_{\sigma^{\prime}, \tau^{\prime}, d} j$ iff $(d, i) \in O_{\sigma^{\prime}}^{\mathrm{obj}},(d, j) \in O_{\tau^{\prime}}^{\mathrm{obj}}$ and $i B_{d} j$. It is easy to see that $B_{\sigma^{\prime}, \tau^{\prime}}$ is a bisimulation between $\sigma^{\prime}$ and $\tau^{\prime}$.

- Suppose $B$ is a bisimulation between $\sigma^{\prime}$ and $\tau^{\prime}$ (not necessarily total or surjective). Suppose $\sigma^{\prime} \sqsubseteq \sigma$ and $\tau^{\prime} \sqsubseteq \tau$. We write $B^{\sigma, \tau}$ for the bisimulation between $\sigma$ and $\tau$ such that, for every $d, B_{d}^{\sigma, \tau}$ has the same graph as $B_{d}$. In other words $B^{\sigma, \tau}$ is $B$, but for the fact that we consider it as a bisimulation between other Sachlagen.

We have $\tau \sqsubseteq \sigma$ iff $\tau=\sigma \mid O_{\tau}^{\text {obj }}$. Moreover, $\tau \sqsubseteq \sigma$ implies that $\tau \preceq \sigma$, since the identical embedding of the sets of indices for an object forms a total bisimulation.

Clearly, $\sigma$ is divided in (possibly zero) non-empty connected parts, the $E_{\sigma}{ }^{-}$ equivalence classes. Some of these $E_{\sigma}$-equivalence classes may be bisimilar to each other. We will see that each $E_{\sigma}$-equivalence class $Y$ corresponds to a Sachverhalt. The classes bisimilar to $Y$ are bisimilar copies of the same Sachverhalt. Here is a first lemma.

Lemma 3.1 Suppose B is a bisimulation between $\sigma$ and $\tau$.

i. The domain of $B^{\circ}$ is closed in $\sigma$ and the range of $B^{\circ}$ is closed in $\tau$. 
ii. Suppose $(d, i)$ is in the domain of $B^{\circ}$. Then, $B_{\sigma[d, i], \tau}^{\circ}: \sigma[d, i] \preceq \tau$.

iii. Suppose $(d, i) B^{\circ}(d, j)$. Then, $B_{\sigma[d, i], \tau[d, j]}^{\circ}: \sigma[d, i] \equiv \tau[d, j]$.

We leave the easy proof to the reader. The following theorem shows that we can decompose $\preceq$ in $\sqsubseteq$ and $\equiv$ in two ways.

Theorem 3.1 We have:

i. $\sigma \preceq \tau$ iff, for some $\sigma^{\prime}, \sigma \equiv \sigma^{\prime}$ and $\sigma^{\prime} \sqsubseteq \tau$.

ii. $\sigma \preceq \tau$ iff, for some $\tau^{\prime}, \sigma \sqsubseteq \tau^{\prime}$ and $\tau^{\prime} \equiv \tau$.

Proof

Ad (i) Suppose $B$ witnesses $\sigma \preceq \tau$. Let $Y$ be the range of $B^{\circ}$. Then, we have: $B_{\sigma, \tau \uparrow Y}: \sigma \equiv \tau \uparrow Y$ and $\tau \uparrow Y \sqsubseteq \tau$. Conversely, if $\sigma \equiv \sigma^{\prime} \sqsubseteq \tau$, then $\sigma \preceq$ $\sigma^{\prime} \preceq \tau$, and, hence, $\sigma \preceq \tau$.

Ad (ii) We define $\tau^{\prime}$ by:

- $\quad I_{\tau^{\prime}, d}:=I_{\sigma, d} \cup\left(\{\sigma\} \times I_{\tau, d}\right)$.

- $\quad(d, l, p) R_{\tau^{\prime}}(e, J, q)$ iff $\left(l \in I_{\sigma, d}\right.$ and $\jmath \in I_{\sigma, e}$ and $(d, l, p) R_{\sigma}$ $(e, J, q))$, or $\left(l=(\sigma, i)\right.$ and $J=(\sigma, j)$ and $\left.(d, i, p) R_{\tau^{\prime}}(e, j, q)\right)$.

We note that $I_{\sigma, d} \cap\left(\{\sigma\} \times I_{\tau, d}\right)$ is empty. Clearly $\sigma \sqsubseteq \tau^{\prime}$.

Suppose $B$ witnesses $\sigma \preceq \tau$. We define $\iota B_{d}^{\star} j$ iff $\left(\iota \in I_{\sigma, d}\right.$ and $\left.\iota B_{d} j\right)$, or $\iota=(\sigma, j)$. Clearly $B^{\star}$ is a total and surjective bisimulation from $\tau^{\prime}$ to $\tau$, so $\tau^{\prime} \equiv \tau$.

\subsection{Sachverhalte}

We remind the reader that a Sachverhalt $\sigma$ is defined as an atom of $\preceq$, that is: $\sigma$ is not $\Perp$ and, whenever $\sigma^{\prime} \preceq \sigma$, then $\sigma^{\prime} \equiv \Perp$ or $\sigma^{\prime} \equiv \sigma$.

Theorem 3.2 We have: $\sigma$ is an atom if and only if $\sigma$ is bisimilar to a non-empty connected sachlage.

Proof Suppose $\sigma$ is an atom. Since $\sigma$ is not the empty Sachlage, there is an object occurrence $(d, i)$ in $\sigma$. Clearly, $\sigma[d, i]$ is non-empty and connected. Moreover, $\sigma[d, i] \preceq \sigma$. Hence, $\sigma \equiv \sigma[d, i]$.

For the converse it is sufficient to show that a non-empty connected sachlage $\sigma$ is an atom. Suppose $B: \sigma^{\prime} \preceq \sigma$ and $\sigma^{\prime}$ is non-empty. Since the range of $B^{\circ}$ must be closed in $\sigma$, it must be equal to $O_{\sigma}^{\mathrm{obj}}$, so $B$ is surjective. Hence $B: \sigma^{\prime} \equiv \sigma$.

Theorem 3.3 Each Sachlage $\sigma$ is the supremum of the Sachverhalte below it. In other words: $\preceq$ is atomistic.

Proof Suppose, for all Sachverhalte $\sigma_{0} \preceq \sigma$, we have $\sigma_{0} \preceq \tau$. It follows that, for each object occurrence $(d, i)$ in $\sigma, \sigma[d, i] \preceq \tau$; say this is witnessed by $B_{(d, i)}$. 
Each $B_{(d, i)}^{\sigma, \tau}$ is a partial bisimulation between $\sigma$ and $\tau$. Clearly the union of the $B_{(d, i)}^{\sigma, \tau}$ will be a total bisimulation between $\sigma$ and $\tau$. Hence $\sigma \preceq \tau$.

\subsection{The Sum-Operation}

We show that every set of Sachlagen has a supremum. Let $\mathcal{S}$ be a set of Sachlagen. We define $\sum \mathcal{S}=: \sigma^{+}$as follows.

- $\quad I_{\sigma^{+}, d}:=\left\{(\sigma, i) \mid \sigma \in \mathcal{S}\right.$ and $\left.i \in I_{\sigma, d}\right\}$.

- $(d,(\sigma, i), p) R_{\sigma^{+}}\left(d^{\prime},\left(\sigma^{\prime}, i^{\prime}\right), p^{\prime}\right): \Leftrightarrow \sigma=\sigma^{\prime}$ and $(d, i, p) R_{\sigma}\left(d^{\prime}, i^{\prime}, p^{\prime}\right)$.

We note that $\sum$ is defined as an operation on sets of Sachlagen, where $\equiv$ is not divided out. We have:

Theorem 3.4 The operation $\sum$ is the supremum operation for our ordering $\preceq$ (modulo $\equiv$ ).

Proof Consider any $\sigma$ in $\mathcal{S}$. We define a bisimulation $B$ from $\sigma$ to $\sigma^{+}$as follows. Let $i$ be in $I_{d}$. We set: $i B_{d}(\sigma, i)$. It is easily seen that $B$ is indeed a total bisimulation. So, $\sigma \preceq \sigma^{+}$.

Suppose $B_{\sigma}: \sigma \preceq \tau$, for each $\sigma \in \mathcal{S}$. We define $(\sigma, i) B_{d}^{+} j: \Leftrightarrow i B_{\sigma, d} j$. It is easily seen that $B^{+}$is a total bisimulation from $\sigma^{+}$to $\tau$. Hence $\sigma^{+} \preceq \tau$.

Note that we do not have enough information yet to construct the maximal Sachlage $\pi$ as the sum of all Sachlagen: we do not yet know that we there is a set of representatives that contains a representative for each Sachlage (as an object modulo bisimulation). We will show that such a set exists in Theorem 4.3.

We are mostly interested in properties of $\sum$ modulo bisimulation, however the following property is dependent on the precise implementation.

Theorem 3.5 Suppose $\mathcal{S} \subseteq \mathcal{S}^{\prime}$. Then, $\sum \mathcal{S} \sqsubseteq \sum \mathcal{S}^{\prime}$

We leave the easy proof to the reader.

We say that a Sachlage $\sigma$ is completely join prime, if, for every set of Sachlagen $\mathcal{S}$, if $\sigma \preceq \sum \mathcal{S}$, then, for some $\sigma^{\prime} \in \mathcal{S}, \sigma \preceq \sigma^{\prime}$.

Theorem 3.6 Every Sachverhalt is completely join prime.

Proof Consider any Sachverhalt $\sigma$. Let $\mathcal{S}$ be a set of Sachverhalte. Suppose $B: \sigma \preceq \sum \mathcal{S}$. Let $(d, i)$ be any object occurrence in $\sigma$ and suppose $i B_{d}\left(\sigma^{\prime}, i^{\prime}\right)$. Remember that $\sigma[d, i]$ is a connected component of $\sigma$ that is bisimilar to $\sigma$. Let $B_{0}$ be a bisimulation from $\sigma$ to $\sigma[d, i]$. We define $B^{\prime}$ between $\sigma$ and $\sigma^{\prime}$, by $j B_{e}^{\prime} j^{\prime}$ iff $j B_{0, e} j_{0}$ and $j_{0} B_{e}\left(\sigma^{\prime}, j^{\prime}\right)$, for some $j_{0} \in I_{\sigma[d, i]}$. It is easy to see that $B^{\prime}$ witnesses that $\sigma \preceq \sigma^{\prime}$. 
We give two definitions.

- $\Theta_{\sigma}$ is the set of $\sigma[d, i]$ such that $(d, i)$ is an object occurrence in $\sigma$. We could also say: $\Theta_{\sigma}$ is the set of connected $\sigma_{0}$ such that $\sigma_{0} \sqsubseteq \sigma$.

- Let $\mathcal{S}$ and $\mathcal{S}^{\prime}$ be sets of Sachlagen. We have: $\mathcal{S} \equiv \mathcal{S}^{\prime}$ iff, for all $\sigma \in \mathcal{S}$, there is a $\sigma^{\prime} \in \mathcal{S}^{\prime}$, such that $\sigma \equiv \sigma^{\prime}$, and for all $\sigma^{\prime} \in \mathcal{S}^{\prime}$, there is a $\sigma \in \mathcal{S}$, such that $\sigma \equiv \sigma^{\prime}$.

A Sachlage functions in many respects as a set of Sachverhalte.

\section{Theorem 3.7 We have:}

i. If $\mathcal{S} \equiv \mathcal{S}^{\prime}$, then $\sum \mathcal{S} \equiv \sum \mathcal{S}^{\prime}$.

ii. $\sigma \equiv \sum \Theta_{\sigma}$.

iii. For any Sachverhalt $\sigma_{0}$ with $\sigma_{0} \preceq \sigma$, there is a $\sigma_{1} \in \Theta_{\sigma}$, such that $\sigma_{0} \equiv \sigma_{1}$.

iv. $\sigma \equiv \sigma^{\prime}$ iff $\Theta_{\sigma} \equiv \Theta_{\sigma^{\prime}}$.

v. $\sigma \equiv \sigma^{\prime}$ iff, for all Sachverhalte $\sigma_{0}, \sigma_{0} \preceq \sigma$ iff $\sigma_{0} \preceq \sigma^{\prime}$.

Proof (i) and (ii) are easy. For (iii), note that, since $\sigma_{0} \preceq \sigma \equiv \sum \Theta_{\sigma}$, we find, that $\sigma_{0} \equiv \sigma_{1}$, for some $\sigma_{1}$ in $\Theta_{\sigma}$, since $\sigma_{0}$ is completely join prime.

We prove (iv). For the right-to-left direction, we use (i) and (ii). For the leftto-right direction, suppose $\sigma \equiv \sigma^{\prime}$ and $\sigma_{0} \in \Theta_{\sigma}$. Then $\sigma_{0} \preceq \sigma^{\prime}$, so by (iii), there is a $\sigma_{0}^{\prime} \in \Theta_{\sigma^{\prime}}$, such that $\sigma_{0} \equiv \sigma_{1}$. Similarly, we prove that if $\sigma_{0}^{\prime} \in \Theta_{\sigma^{\prime}}$, then, for some $\sigma_{0} \in \Theta_{\sigma}, \sigma_{0}^{\prime} \equiv \sigma_{0}$. Hence $\Theta_{\sigma} \equiv \Theta_{\sigma^{\prime}}$.

We prove $(\mathrm{v})$. From left to right is trivial. Suppose, for all Sachverhalte $\sigma_{0}$, $\sigma_{0} \preceq \sigma$ iff $\sigma_{0} \preceq \sigma^{\prime}$. Then, each element $\sigma_{0}$ of $\Theta_{\sigma}$ is below $\sigma^{\prime}$. Ergo, $\sigma \equiv \sum \Theta_{\sigma} \preceq$ $\sigma^{\prime}$. Similarly, $\sigma^{\prime} \preceq \sigma$.

\subsection{The Conjunction-Operation}

We can define an operation on Sachlagen that produces an infimum in several ways. We take one such way as our official choice. For some discussion of the options, see Remark 3.10.

We define the conjunction $\sigma \otimes \tau$ of Sachlagen $\sigma$ and $\tau$ as follows. Consider the maximal bisimulation $B$ between $\sigma$ and $\tau$. Note that $B$ need not be total or surjective; it might even be empty. Let $X$ be the domain of $B^{\circ}$. We take $\sigma \otimes \tau:=\sigma \uparrow X$.

Theorem 3.8 $\sigma_{0} \oslash \sigma_{1}$ is, modulo $\equiv$, the infimum of $\sigma_{0}$ and $\sigma_{1}$.

Proof We will use our convention that for any bisimulation $B^{\prime}$, the domain of $B^{\prime}$ is the domain of $B^{\prime \circ}$.

Let $B$ be the maximal bisimulation from $\sigma_{0}$ to $\sigma_{1}$.

Note that $\left(\sigma_{0} \otimes \sigma_{1}\right) \sqsubseteq \sigma_{0}$, and hence, $\left(\sigma_{0} \oslash \sigma_{1}\right) \preceq \sigma_{0}$. Moreover, $B_{\sigma \bowtie \tau, \tau}:(\sigma \otimes$ $\tau) \preceq \tau$.

Suppose $C_{i}: \tau \preceq \sigma_{i}$, for $i=0,1$. We note that $C:=\breve{C}_{0} ; C_{1}$ is a bisimulation from $\sigma_{0}$ to $\sigma_{1}$. The domain $C$ of is the domain of $\breve{C}_{0}$, since $C_{1}$ is total. The 
domain of $\breve{C}_{0}$ is the range of $C_{0}$. We may conclude that the range of $C_{0}$ is included in the domain of $B$, since $B$ is maximal. Ergo: $C_{0, \tau, \sigma_{0} \otimes \sigma_{1}}: \tau \preceq$ $\left(\sigma_{0} \otimes \sigma_{1}\right)$.

We prove distributivity of $₫$ over $\sum$.

Theorem 3.9 We have $\sigma \otimes \sum \mathcal{S} \equiv \sum\left\{\sigma \otimes \sigma^{\prime} \mid \sigma^{\prime} \in \mathcal{S}\right\}$.

Proof Let $\sigma_{0}$ be a Sachverhalt. We have:

$$
\begin{aligned}
\sigma_{0} \preceq\left(\sigma \otimes \sum \mathcal{S}\right) & \Leftrightarrow \sigma_{0} \preceq \sigma \text { and } \sigma_{0} \preceq \sum \mathcal{S} \\
& \Leftrightarrow \sigma_{0} \preceq \sigma \text { and, for some } \sigma^{\prime} \in \mathcal{S}, \sigma_{0} \preceq \sigma^{\prime} \\
& \Leftrightarrow \text { for some } \sigma^{\prime} \in \mathcal{S}, \sigma_{0} \preceq \sigma \text { and } \sigma_{0} \preceq \sigma^{\prime} \\
& \Leftrightarrow \text { for some } \sigma^{\prime} \in \mathcal{S}, \sigma_{0} \preceq\left(\sigma \otimes \sigma^{\prime}\right) \\
& \Leftrightarrow \sigma_{0} \preceq \sum\left\{\sigma \otimes \sigma^{\prime} \mid \sigma^{\prime} \in \mathcal{S}\right\}
\end{aligned}
$$

The desired conclusion follows from Theorem 3.7(v)

Remark 3.10 There are several alternative choices for our operation $₫$. Perhaps the two most obvious are:

- $\sigma \otimes \tau:=\sum\left\{\sigma^{\prime} \sqsubseteq \sigma \mid \sigma^{\prime} \preceq \tau\right\}$.

- $\sigma \otimes \tau:=\sum\left\{\sigma^{\prime} \sqsubseteq \sigma \mid \sigma^{\prime}\right.$ is a Sachverhalt and $\left.\sigma^{\prime} \preceq \tau\right\}$.

Note that in both cases, we really do our summation over a set.

A third alternative is in in the spirit of our chosen definition of $\varnothing$ and connects this to a beautiful observation: bisimulations can themselves be considered as Sachlagen. Consider any bisimulation $C$ between $\sigma$ and $\tau$. We define a Sachlage $\Phi(C)$ by:

- $\quad I_{\Phi(C), d}:=\left\{(i, j) \mid i C_{d} j\right\}$,

- $\quad(d,(i, j), p) R_{\Phi(C)}\left(e,\left(i^{\prime}, j^{\prime}\right), q\right)$ iff $(d, i, p) R_{\sigma}\left(e, i^{\prime}, q\right)$.

Note that it follows that:

$(d,(i, j), p) R_{\Phi(C)}\left(e,\left(i^{\prime}, j^{\prime}\right), q\right)$ iff $(d, j, p) R_{\tau}\left(e, j^{\prime}, q\right)$.

We can now easily show that $\Phi(C) \preceq \sigma$ and $\Phi(C) \preceq \tau$. Moreover, whenever $C_{0}: v \preceq \sigma$ and $C_{1}: v \preceq \tau$, then $\Phi\left(\breve{C}_{0} ; C_{1}\right) \equiv v$.

Clearly $C^{\circ} \subseteq C^{\prime \circ}$ implies $\Phi(C) \sqsubseteq \Phi\left(C^{\prime}\right)$. This suggests the following definition of $\oslash$. We define $\sigma \otimes \tau:=\Phi(B)$, where $B$ is the maximal bisimulation between the Sachlagen $\sigma$ and $\tau$.

\subsection{The Sachlagen form a Set}

We assumed that all the ingredients of what makes a logical space $(D, T, M$, $P, F)$ are just sets. It follows that any Sachlage can be modeled as a set. 
However the totality of the Sachlagen is a proper class, since we have put no constraint on the choice of the occurrences. Since, the sum of arbitrarily many copies of a Sachlage is again a Sachlage, we would even obtain a proper class if we could pick an element of every isomorphism type of the Sachlagen.

In Section 4, we will produce a unique normal form modulo bisimulation for each Sachlage. As we will see these normal forms form a set. Hence the Sachlagen form a set modulo bisimulation (Theorem 4.3). As a consequence of this result, we find:

Theorem 3.11 In any logical space, we have a maximal Sachlage $\pi$. The Sachlagen modulo bisimulation ordered by $\preceq$ form a atomistic distributive complete lattice.

Remark 3.12 We can also define subtraction of Sachlagen, making the Sachlage modulo bisimulation into a Boole Algebra.

We note that this Boole algebra is the Boole algebra of Sachlagen in their inclusive role. It is not the Boole Algebra that Wittgenstein defined over logical space. Wittgenstein's algebra is defined on sets of Sachlagen in their exclusive role. A Sachlage $\sigma$ in the inclusive role reappears as a set of Sachlagen in their exclusive role as $\uparrow \sigma:=\{\tau \mid \sigma \preceq \tau\}$. ${ }^{11}$ Our sum corresponds to Wittgenstein's conjunction: $\uparrow \sum\{\sigma, \tau\}:=\uparrow \sigma \cap \uparrow \tau$.

\section{Normal Forms for Sachlagen}

In this section, we provide three kinds of normal form for Sachlagen. The last two kinds make a bisimulation-free approach to Sachlagen possible. The first two kinds of normal forms are the usual normal forms familiar from e.g. modal logic: bisimulation collapse and canonical unraveling. The last kind, canonical bisimulation collapse, consists of the bisimulation collapses of the canonical unravelings. Since, our Sachlagen are more constrained than most kinds of transition systems the normal forms are, in a sense, better. E.g., for canonical unraveling in modal logic one needs to choose a cardinal. This is unnecessary when one studies graded modalities and employs counting bisimulation. Sachlagen and bisimulation on Sachlagen are more like graded transition systems and counting bisimulation. (See e.g. [3]).

\subsection{Bisimulation Collapse}

We can find a minimal representation of a Sachlage $\sigma:=(I, R)$ as follows. Let $\mathcal{B}$ be the set of all bisimulations between $\sigma$ and itself. This set is not empty,

\footnotetext{
${ }^{11}$ I am ignoring the fact that we do not have a set here. We clearly can tell a story, e.g. using the normal forms of the next section, to make sense of this.
} 
since $\mathrm{id}_{\sigma}$ is in it. Let $B^{\dagger}:=\bigcup \mathcal{B}$. So $B^{\dagger}$ is the maximal bisimulation of $\sigma$ with itself. By Theorem 2.3, each $B_{d}^{\dagger}$ is an equivalence relation. We write $[i]_{B_{d}^{\dagger}}$ for the $B_{d}^{\dagger}$-equivalence class of $i$ in $I_{d}$. We define coll $(\sigma):=\sigma^{\dagger}$ by:

- $I_{d}^{\dagger}:=I_{d} / B_{d}^{\dagger}$,

- $\left(d,[i]_{B_{d}^{\dagger}}, p\right) R^{\dagger}\left(e,[j]_{B_{e}^{\dagger}}, q\right): \Leftrightarrow \exists i_{0} \in[i]_{B_{d}^{\dagger}}, j_{0} \in[j]_{B_{e}^{\dagger}}\left(d, i_{0}, p\right) R\left(e, j_{0}, q\right)$.

It is easy to see that $\sigma^{\dagger}$ is indeed a Sachlage and that $\sigma^{\dagger} \equiv \sigma$. Moreover, we find that, whenever $\sigma_{0} \equiv \sigma_{1}$, then $\sigma_{0}^{\dagger}$ and $\sigma_{1}^{\dagger}$ are isomorphic.

In the next subsection, we will develop normal forms using canonical unraveling. These normal forms are unique simpliciter. As soon as we have these, the bisimulation collapse of such a canonical unraveling is a normal form that is both bisimulation-minimal and uniquely determined for each Sachlage.

\subsection{The Canonical Unraveling}

Consider a logical space. We consider (non-empty) sequences $f$ of the form

$$
\left(d_{0}, p_{01}, p_{10}, d_{1}, p_{11}, p_{20}, d_{2}, \ldots, p_{n 0}, d_{n}\right) .
$$

Here $p_{01}$ is in $P_{d_{0}}$, and, for $k>0, p_{k 0}$ and $p_{k 1}$ are in $P_{d_{k}}$ and $p_{k 0} \neq p_{k 1}$. Moreover we demand: $F_{d_{k}}\left(p_{k 1}\right) M F_{d_{k+1}}\left(p_{(k+1) 0)}\right.$. We call these sequences paths. The length of a path is the unique $n$ such that the length of the path qua sequence is $3 n+1$. So, a path of length 0 has the form $(d)$.

Since the objects form a set and the poles of an object form a set, the paths form a set.

We define:

- $\quad f$ \# $g$ iff, for some $d$, both $f$ and $g$ start from $d$, and either $f=(d)$ or $g=$ $(d)$ or $\left(f=(d, p, \ldots)\right.$ and $g=\left(d, p^{\prime}, \ldots\right)$ and $\left.p \neq p^{\prime}\right)$.

- $\quad \breve{f}$ is the result of reading $f$ backwards.

- $f \star g$ is defined if $\breve{f} \# g$. If our operation is defined, we have:

$$
\overbrace{(\ldots, d)}^{f} \star \overbrace{(d, \ldots)}^{g}=(\ldots, d, \ldots) .
$$

We note that the result is again a path.

The paths with $\star$ form a partial monoid with as units the paths $(d)$. Warning: the paths do not generally form a category. E.g., $(d, p, e) \star(e) \star(e, p, d)$ is not defined, even if both $(d, p, e) \star(e)$ and $(e) \star(e, p, d)$ are.

A set $U$ of paths is coherent iff, for some object $d$,

i. $U$ contains the path $(d)$.

ii. All elements of $U$ have starting point (domain) $d$. 
iii. For any $f$ and $g$ in $U$, there are $h, f_{0}, g_{0}$, such that $f=h \star f_{0}, g=h \star$ $g_{0}$ and $f_{0} \# g_{0}$. In other words, either one of the paths (weakly) extends the other, or, after sharing an initial part they diverge by taking different poles. $^{12}$

iv. $U$ is closed under initial subpaths.

v. Suppose $f=(\ldots, q, e)$ is in $U$ and $r$ is a pole of $e$ unequal to $q$. Then, there are $r^{\prime}$ and $e^{\prime}$, such that $f \star\left(e, r, r^{\prime}, e^{\prime}\right)$ is in $U$. In other words, when a path can be extended, it is extended in $U$.

We note that, the totality of the coherent sets of a given space forms a set.

A coherent set is something like a map that a fairy living in a Sachlage would make of the Sachlage, assuming that the fairy would assume the Sachlage to be stable, in the sense that backtracking leads to the same places, and that the fairy would never unnecessarily identify places.

Consider any coherent set $U$. We associate a Sachverhalt $\operatorname{svh}(U):=\sigma_{U}$ to $U$ in the following manner. We define:

- $I_{\sigma_{U}, e}:=\{f \in U \mid \operatorname{cod}(f)=e\}$,

- $\quad(e, f, q) R_{\sigma_{U}}\left(e^{\prime}, f^{\prime}, q^{\prime}\right)$ iff $f=f^{\prime} \star\left(e^{\prime}, q^{\prime}, q, e\right)$ or $f^{\prime}=f \star\left(e, q, q^{\prime}, e^{\prime}\right)$.

We easily see that $\sigma_{U}$ is a Sachverhalt. Since the coherent sets form a set, also the $\sigma_{U}$ form a set.

Theorem 4.1 Given any Sachlage $\sigma$ and any object occurrence $(d, i)$ in $\sigma$, we can find a coherent set $\operatorname{cs}(\sigma,(d, i)):=U_{\sigma,(d, i)}$, and a functional bisimulation $G$ (or, more explicitly, $\left.G_{\sigma,(d, i)}\right)$ from $\operatorname{svh}(\operatorname{cs}(\sigma,(d, i)))$ to $\sigma$, such that $G((d,(d))=$ $(d, i)$. Moreover, for any bisimulation $B$ from $\operatorname{svh}(\operatorname{cs}(\sigma,(d, i)))$ to $\sigma$ with $(d,(d)) B^{\circ}(d, i)$, we have $G^{\circ} \subseteq B^{\circ}$.

Proof We define $U_{\sigma,(d, i)}$ as follows. By recursion on path length we simultaneously define elements of $U$ and a mapping $G_{0}$ on these elements such that $G_{0}(f) \in I_{\operatorname{cod}(f)}$. We put $(d)$ in $U$ and set $G_{0}((d)):=i$. We treat the case that $f=(\ldots, q, e)$ and $G_{0}(f)=: j$ are already given. The case starting from $(d)$ is similar. Suppose $q^{\prime} \in I_{e} \backslash\{q\}$. Then, for some unique $j^{\prime}, q^{\prime \prime}, e^{\prime}$, we have $\left(e, j, q^{\prime}\right) R_{\sigma}\left(e^{\prime}, j^{\prime}, q^{\prime \prime}\right)$. We add $f^{\prime}:=f \star\left(e, q^{\prime}, q^{\prime \prime}, e^{\prime}\right)$ to $U$, and take $G_{0}\left(f^{\prime}\right):=j^{\prime}$.

We take $(e, f) G\left(e, G_{0}(f)\right)$, for $e=\operatorname{cod}(f)$. It is not difficult to see that $G$ is a functional bisimulation from $\operatorname{svh}(\operatorname{cs}(\sigma,(d, i)))$ to $\sigma$. That $G^{\circ}$ is part of any bisimulation from $\operatorname{svh}(\operatorname{cs}(\sigma,(d, i)))$ to $\sigma$ that connects $(d,(d))$ to $(d, i)$ follows with an easy induction on path length.

\footnotetext{
${ }^{12}$ We note that this could also be formulated as follows: consider two different paths $f$ and $g$ in $U$. Let $k$ be the smallest number such that either $\left((f)_{k}\right.$ is defined and $(g)_{k}$ is undefined) or $\left((f)_{k}\right.$ is undefined and $(g)_{k}$ is defined) or $\left((f)_{k}\right.$ and $(g)_{k}$ are both defined and different), then $k$ is $3 k_{0}+1$, for some $k_{0}$ (assuming that we start counting with 0 ).
} 
Theorem 4.2 Suppose $\sigma$ is a Sachverhalt and $(d, i)$ is an object-occurrence in $\sigma$, then $\sigma \equiv \operatorname{svh}(\operatorname{cs}(\sigma,(d, i)))$.

Proof Since, $G_{\sigma,(d, i)}$ is surjective to $\sigma[d, i]$ and since $\sigma$ is a Sachverhalt, we find $\sigma \equiv \operatorname{svh}(\operatorname{cs}(\sigma,(d, i)))$.

Thus, we see that all Sachverhalte are bisimilar to $\sigma_{U}$, for some coherent $U$. We are now in the position to give our canonical unraveling of $\sigma$. This uses, of course, that the $\sigma_{U}$ form a set.

- $\operatorname{unr}(\sigma):=\sum\left\{\sigma_{U} \mid U\right.$ is coherent and $\left.\sigma_{U} \preceq \sigma\right\}$.

\section{Theorem 4.3 We find:}

a. For each Sachlage $\sigma$, $\operatorname{unr}(\sigma) \equiv \sigma$.

b. If $\sigma \equiv \tau$, then $\operatorname{unr}(\sigma)=\operatorname{unr}(\tau)$.

c. If $\sigma \preceq \tau$, then $\operatorname{unr}(\sigma) \sqsubseteq \operatorname{unr}(\tau)$.

d. The canonical unravelings unr $(\sigma)$ form a set.

Proof Our ordering is atomistic and every atom is bisimilar to a $\sigma_{U}$. Hence, $\operatorname{unr}(\sigma) \equiv \sigma$. This gives us (a). Moreover, (b) is trivial, and (c) is immediate from Theorem 3.5. Finally, since the $\sigma_{U}$ form a set, say $\mathcal{X}$, the subsets of $\mathcal{X}$ form a set. Hence, the objects of the form $\operatorname{unr}(\sigma)$ form a set.

We note that our earlier result that the Sachlagen modulo bisimulation form a distributive atomistic complete lattice follows immediately from Theorem 4.3 , since $\sqsubseteq$ is essentially the subset relation.

The material we developed up to this point is sufficient for the purposes of our normal form theorem. However, there is a bit more to say about our construction. We have the following theorem.

Theorem 4.4 Let $U$ be a coherent set starting with $(d)$. Suppose $B$ is a bisimulation from $\sigma_{U}$ to $\sigma$ with $(d,(d)) B^{\circ}(d, i)$. Then $U=\operatorname{cs}(\sigma,(d, i))$ and $G_{\sigma,(d, i)}^{\circ} \subseteq B^{\circ}$.

Proof Let $G:=G_{\sigma,(d, i)}$ and let $G_{0}$ be the corresponding function of paths. We show by induction on path length that $W:=\operatorname{cs}(\sigma,(d, i))$ is a subset of $U$ and $f B G_{0}(f)$.

Clearly, (d) is in $U$ and (d) $B i$.

Suppose $(d, p, q, e)$ is in $W$ and $G_{0}((d, p, q, e))=j$. Then, we have $(d, i, p) P_{\sigma}(e, j, q)$. Since $(d,(d)) B^{\circ}(d, i)$, we find that $(d,(d), p) R_{\sigma_{U}}(e, h, q)$ and $h B j$, for some $h$. The only possible such $h$ is $(d, p, q, e)$. So $(d, p, q, e)$ is in $U$ and $(d, p, q, e) B j$.

Suppose we have the induction hypothesis for $(\ldots, q, e)$. Let $G((\ldots, q, e))=$ $j$. Suppose $\left(\ldots, q, e, p^{\prime}, q^{\prime}, e^{\prime}\right)$ is in $W$ and $G_{0}\left(\left(\ldots, q, e, p^{\prime}, q^{\prime}, e^{\prime}\right)\right)=j^{\prime}$. Since, $(e,(\ldots, q, e)) B^{\circ}(e, j)$, we find that $(e,(\ldots, q, e)) R_{\sigma_{U}}\left(e^{\prime}, h^{\prime}, p^{\prime}\right)$ and $h^{\prime} B j^{\prime}$, 
for some $h^{\prime}$. Since $p^{\prime} \neq q, h^{\prime}$ must be an extension of $(\ldots, q, e)$. Ergo, $h^{\prime}=$ $\left(\ldots, q, e, p^{\prime}, q^{\prime}, e^{\prime}\right)$. So, $\left(\ldots, q, e, p^{\prime}, q^{\prime}, e^{\prime}\right)$ is in $U$ and $\left(\ldots, q, e, p^{\prime}, q^{\prime}, e^{\prime}\right) B j^{\prime}$.

We may conclude that $W \subseteq U$. It is immediate that $W=U$.

As an immediate corollary we have:

Theorem 4.5 If $U$ is a coherent set starting with $(d)$, then $U=$ $\operatorname{cs}(\operatorname{svh}(U),(d,(d)))$.

Remark 4.6 The reader who has some knowledge of category theory, will suspect there is an adjunction here. That is indeed the case. Consider the category with as objects the pointed Sachlagen $(\sigma,(d, i))$ and as morphisms between $(\sigma,(d, i))$ and $(\tau,(e, j))$ the bisimulations $B$ (not necessarily total or surjective) such that $(d, i) B^{\circ}(e, j)$ (and, hence, $d=e$ ). Consider as second category the coherent sets with as morphisms just the identity. Then $\mathrm{svh}^{+}$is the left adjoint of cs, where $\operatorname{svh}^{+}(U):=(\operatorname{svh}(U),(d,(d))$ for $(d)$ the starting point of $U$.

Our normal forms still 'contain' a number of bisimilar copies of a Sachverhalt, since $\sigma_{U} \equiv \sigma_{V}$, does not imply $\sigma_{U}=\sigma_{V}$. We show that the situation is a bit better: $\sigma_{U} \equiv \sigma_{V}$, does imply $\sigma_{U}$ is isomorphic to $\sigma_{V}$. So, for each Sachverhalt, our normal form contains only isomorphic copies.

Theorem 4.7 Suppose $\sigma_{U} \equiv \sigma_{V}$. Then, $\sigma_{U}$ is isomorphic to $\sigma_{V}$.

Proof Suppose $B$ witnesses that $\sigma_{U}$ is bisimilar to $\sigma_{V}$. Suppose $U$ starts with $(d)$ and $(d) B h$. By Theorem 4.4, we find that $U=\operatorname{cs}\left(\sigma_{V},(d, h)\right)$ and $G:=$ $G_{\sigma_{V},(d, h)}$ is a functional sub-bisimulation of $B$.

Suppose that $V$ starts with $(e)$. It is easily seen that $\breve{h} G(e,(e))$. Applying Theorem 4.4 to $\sigma_{V}$ and $\breve{G}$, we find that $V=\operatorname{cs}(U,(e, \breve{h}))$. Let $G^{\prime}:=G_{\sigma_{V},(e, \breve{h})}$. We note that $G ; G^{\prime}$ is a functional bisimulation from $\sigma_{U}$ to $\sigma_{U}$. Moreover, $(d)\left(G ; G^{\prime}\right)(d)$. Applying Theorem 4.4 for a third time, we find that $G$; $G^{\prime}$ is the identity bisimulation of $\sigma_{U}$. Similarly, $G^{\prime} ; G$ is the identity bisimulation of $\sigma_{V}$. We may conclude that $\sigma_{U}$ and $\sigma_{V}$ are isomorphic.

\subsection{The Canonical Collapse}

The canonical collapse is obtained by collapsing the canonical unraveling. Thus, we define the canonical collapse of $\sigma$ by:

- $\quad \operatorname{C-coll}(\sigma):=\operatorname{coll}(\operatorname{unr}(\sigma))$.

This collapse is evidently bisimulation minimal. Moreover, we have: if $\sigma \equiv \tau$, then $\mathrm{C}$-coll $(\sigma)=\mathrm{C}$-coll $(\tau)$. So each $\equiv$-equivalence class has a unique normal form. All this is trivial by the preceding results on coll and unr. However, we also have the following theorem. 
Theorem 4.8 Suppose $\sigma \preceq \tau$. Then, c-coll $(\sigma) \sqsubseteq \mathrm{c}-\operatorname{coll}(\tau)$.

Proof Suppose $\sigma \preceq \tau$. Let $B$ be the maximal bisimulation on $\operatorname{unr}(\sigma)$ and let $C$ be the maximal bisimulation on $\operatorname{unr}(\tau)$.

Consider $\imath$ in $I_{\mathrm{c}-\mathrm{coll}(\sigma), d}$. The occurrence $\iota$ is of the form $\left[\left(\sigma_{U}, f\right)\right]_{B_{d}}$, where $\left(d,\left(\sigma_{U}, f\right)\right)$ is an object-occurrence in $\operatorname{unr}(\sigma)$, and, hence, $\sigma_{U} \preceq \sigma$.

Clearly, $\left(d,\left(\sigma_{U}, f\right)\right)$ is also an object-occurrence in $\operatorname{unr}(\tau)$. It follows that the occurrence $\left[\left(\sigma_{U}, f\right)\right]_{C_{d}}$ is in $I_{\mathrm{c}-\operatorname{coll}(\tau), d}$. So it is sufficient to show that $\left[\left(\sigma_{U}, f\right)\right]_{C_{d}}=\left[\left(\sigma_{U}, f\right)\right]_{B_{d}}$.

Suppose $\left(\sigma_{U}, f\right) C_{d}\left(\sigma_{V}, g\right)$. It follows, by Lemma 3.1(iii), that the Sachverhalte $\widetilde{\sigma}_{U}:=(\operatorname{unr}(\tau))\left[\left(d,\left(\sigma_{U}, f\right)\right)\right]$ and $\widetilde{\sigma}_{V}:=(\operatorname{unr}(\tau))\left[\left(d,\left(\sigma_{V}, g\right)\right)\right]$ are bisimilar. But, $\widetilde{\sigma}_{U}$ is an isomorphic copy of $\sigma_{U}$ and $\widetilde{\sigma}_{V}$ is an isomorphic copy of $\sigma_{V}$. Ergo, $\sigma_{U}$ is bisimilar to $\sigma_{V}$, and, hence, $\sigma_{V} \preceq \sigma$. It follows that $\left(d,\left(\sigma_{V}, g\right)\right)$ is an object-occurrence in unr $(\sigma)$. Since, $\left(d,\left(\sigma_{U}, f\right)\right)$ and $\left(d,\left(\sigma_{V}, g\right)\right)$ are bisimilar according to $C^{\circ}$ restricted to unr $(\sigma)$ and since $B$ is maximal on unr $(\sigma)$, we find $\left(d,\left(\sigma_{U}, f\right)\right) B^{\circ}\left(d,\left(\sigma_{V}, g\right)\right)$. We may conclude that $\left(\sigma_{V}, g\right) \in\left[\left(\sigma_{U}, f\right)\right]_{B_{d}}$. Hence, $\left[\left(\sigma_{U}, f\right)\right]_{C_{d}}=\left[\left(\sigma_{U}, f\right)\right]_{B_{d}}$, as desired.

Thus the order structure takes a very simple form on canonical collapse normal forms.

\section{Perspectives}

In this section, we briefly touch on possible variations, improvements and extensions of the framework.

\subsection{Configurations}

What kind of structures do we get when we drop the demand of saturatedness? Let's call these structures configurations. A configuration $\gamma$ is defined as follows.

- A function $I$ from objects to (possibly empty) sets of indices.

- A binary relation $R$ on $O_{\gamma}^{\text {pole }}$, where $O_{\gamma}^{\text {pole }}$, the set of pole occurrences, is the set of $(d, i, p)$, where $i$ is in $I_{d}$ and $p$ is in $P_{d}$. We demand that $R$ is functional and symmetrical and that $(d, i, p) R\left(d^{\prime}, i^{\prime}, p^{\prime}\right)$ implies $F_{d}(p) M$ $F_{d^{\prime}}\left(p^{\prime}\right)$.

So a configuration is a Sachlage minus totality. We define:

- $\quad P_{\gamma}:=\left\{(d, i, p) \in O_{\gamma}^{\text {pole }} \mid(d, i, p) \notin \operatorname{dom}\left(R_{\gamma}\right)\right\}$.

- $F_{\gamma}(d, i, p):=F_{d}(p)$.

Note that with every object $d$ there corresponds a configuration $\llbracket d \rrbracket$ given by (i) $I_{d^{\prime}}:=\emptyset$, if $d^{\prime} \neq d$, and $I_{d^{\prime}}:=\{0\}$, if $d^{\prime}=d$, and (ii) $R$ is the empty. 
We define a simulation $B$ from $\gamma$ to $\gamma^{\prime}$ just as we defined a bisimulation before, now only asking for the forward property. We define a bisimulation as before. We define $\gamma \preceq \gamma^{\prime}$ iff there is a total simulation from $\gamma$ to $\gamma^{\prime}$. We count $\gamma$ and $\gamma^{\prime}$ the same iff there is a total and surjective bisimulation between them.

Note that, on Sachlagen our new $\preceq$ coincides with our old $\preceq$. However, on Sachlagen the induced equivalence relation of $\preceq$ coincided with the presence of a total and surjective bisimulation. This is not true anymore for configurations.

We note that we can define the existence of a configuration $\gamma$ as the fact that $\gamma \preceq \sigma$, for the maximal existing Sachlage $\sigma$. We can say that an object $d$ exists if $\llbracket d \rrbracket$ does. Thus, the fixed store of objects in the tractarian universe can be best conceived of as a store of possible objects, that come into existence if they occur in some fact.

Associated with configurations, there is a lingering doubt: is the framework we developed a reflective equilibrium? There is a feeling that configurations are something like complex objects. E.g., when we think about a morphism between logical spaces, we could imagine mapping objects to configurations. However, the poles of a configuration $\gamma$ do not quite behave as the poles of objects. If we multiply the poles of an object it is always in a coordinated way: we copy the whole set. However, if we take a bisimulation variant of a configuration, it is clear that some groups of poles will be linked in multiplication or contraction, but some groups are not. Can we generalize the notion of object in such a way that objects and configurations behave in the same way?

\subsection{Argument Places}

As we already noted, we implement the most straightforward idea of what the places are. It is clear that we can build in refinements of this treatment rather easily. E.g., on each set of poles $P_{d}$ we can have a group of permutations $G_{d}$. Perhaps we can improve the notion of bisimulation by taking $B$ as a function on objects that assigns to each $d$ a ternary relation $B_{d}$ between $I_{d}$ and $G_{d}$ and $I_{d}^{\prime}$, such that $B_{d}$ satisfies the zig- and the zag-property:

- If $i_{0} B_{d_{0}}^{g_{0}} i_{0}^{\prime}$ and $\left(d_{0}, i_{0}, p_{0}\right) R\left(d_{1}, i_{1}, p_{1}\right)$, then, for some $i_{1}^{\prime}$ in $I_{d_{1}}^{\prime}$ and $g_{1}$ in $G_{d_{1}}$, we have $i_{1} B_{d_{1}}^{g_{1}} i_{1}^{\prime}$ and $\left(d_{0}, i_{0}^{\prime}, g_{0} p_{0}\right) R^{\prime}\left(d_{1}, i_{1}^{\prime}, g_{1} p_{1}\right)$ (zig-property),

- If $i_{0} B_{d_{0}}^{g_{0}} i_{0}^{\prime}$ and $\left(d_{0}, i_{0}^{\prime}, p_{0}\right) R^{\prime}\left(d_{1}, i_{1}^{\prime}, p_{1}\right)$, then, for some $i_{1}$ in $I_{d_{1}}$ and $g_{1}$ in $G_{d_{1}}$, we have $i_{1} B_{d_{1}}^{g_{1}} i_{1}^{\prime}$ and $\left(d_{0}, i_{0}, g_{0}^{-1} p_{0}\right) R\left(d_{1}, i_{1}, g_{1}^{-1} p_{1}\right)$ (zag-property).

I do not know whether this works. Also, I do not think that we can treat all examples produced by Kit Fine in this way.

\subsection{Space and Time}

How do time and space fit into a Wittgensteinean universe? One option would be to take them to be external to the Sachverhalte. However, this has several problems. First it is hard to see how it coheres with 'the world being all that 
is the case'. It is hard to swallow that temporal and spatial relations would be outside the world, part of the unspeakable, so to say. Secondly, if we refuse to put constraints on how subsequent snapshots of the world cohere, then it difficult to understand how one could seriously speak of time at all. There's just nothing connecting the moments.

If we put spatio-temporal locations as objects inside the Sachlage, this gives us the usual problems about how to model, e.g., temporal order. Moreover, consider John is angry. It is plausible that John will have a temporal location argument place and that angry will carry one too too. But inside the Sachverhalt we would like these places to be filled with the same location. The present framework does not seem to have means to enforce this idea. Again, it would be good to find an appropriate enrichment.

Acknowledgements I thank Jaap van der Does, Leon Geerdink, Jesse Mulder, Vincent van Oostrom, Martin Stokhof, Göran Sundholm, and the members of the Utrecht Tractatus Reading Group for their comments and criticisms. I am grateful to the anonymous referee for his/her helpful suggestions. For an acknowledgement of priority to Alan Bawden, see Section 1.6.

Open Access This article is distributed under the terms of the Creative Commons Attribution Noncommercial License which permits any noncommercial use, distribution, and reproduction in any medium, provided the original author(s) and source are credited.

\section{References}

1. Aczel, P. (1988). Non-well-founded sets. CSLI lecture notes (Vol. 14). Stanford: CSLI.

2. Black, M. (1971). A companion to Wittgenstein's Tractatus. Cambridge: Cambridge University Press.

3. D'Agostino, G., \& Visser, A. (2002). Finality regained: A coalgebraic study of Scott-sets and multisets. Archive for Mathematical Logic, 41, 267-298.

4. Fine, K. (2000). Neutral relations. The Philosophical Review, 109, 1-33.

5. Leo, J. (2008). The identitity of argument-places. The Review of Symbolic Logic, 1(3), 335-354.

6. Leo, J. (2008). Modeling relations. Journal of Philosophical Logic, 37, 353-385.

7. Marion, M. (2004). Ludwig Wittgenstein, Introduction au "Tractatus logico-philosophicus". Philosophies. Presses Universitaires de France, Paris.

8. Rieger, A. (2000). An argument for Finsler-Aczel set theory. Mind, 109(434), 241-253.

9. Wittgenstein, L. (1961). Tractatus logico-philosophicus. London: Routledge \& Kegan Paul. 